\title{
Serial assessment of left ventricular diastolic function after Fontan procedure
}

\author{
Y F Cheung, D J Penny, A N Redington
}

\begin{abstract}
Objective-To assess longitudinal changes in systemic ventricular diastolic function late after the Fontan procedure.

Design and patients-Prospective study of 13 patients at 2.8 (2.0) years (early) and again at 11.4 (2.0) years (late) after the Fontan procedure by Doppler echocardiography with simultaneous ECG, phonocardiogram, and respirometer.

Setting-Tertiary paediatric cardiac centre.

Results-The isovolumic relaxation time (IVRT) was significantly longer, and E wave deceleration time, $\mathrm{E}$ and $\mathrm{A}$ wave velocities, and $\mathrm{E}$ :A velocity ratio were reduced compared to normal both early and late after the procedure. The mean (SD) z score of IVRT decreased significantly from $+2.50(1.00)$ to $+1.24(0.80)(\mathrm{p}=0.002)$, and the $\mathrm{z}$ score of the $\mathrm{E}$ wave deceleration time decreased from $-1.69(1.31)$ to $-2.40(1.47)(\mathrm{p}=0.03)$ during follow up. The A wave deceleration time also tended to decrease (early 80 (12) ms $v$ late $73(11) \mathrm{ms}, \mathrm{p}=0.13$ ) with increased follow up. There were no changes of the $\mathrm{E}$ and $\mathrm{A}$ wave velocities and $\mathrm{E}$ :A velocity ratio. The $\mathrm{E}$ wave velocity was inversely related to IVRT both early $(r=-0.82, \mathrm{p}=0.001)$ and late $(r=-0.59$, $\mathrm{p}=0.034)$ after the operation. The prevalence of diastolic flow during isovolumic relaxation decreased from $85 \%(11 / 13)$ to $38 \%(5 / 13)(\mathrm{p}=0.04)$, while that of mid diastolic flow increased from $23 \%(3 / 13)$ to $77 \%(10 / 13)(p=0.02)$ between the two assessments.

Conclusions-Left ventricular diastolic function remains highly abnormal late after the Fontan procedure. The longitudinal changes demonstrated on follow up are compatible with reduction of left ventricular compliance in addition to persisting abnormalities of relaxation.

(Heart 2000;83:420-424)
\end{abstract}

Keywords: diastolic function; Fontan procedure

A progressive deterioration in functional status occurs during long term follow up of patients after the Fontan procedure. ${ }^{1-3}$ The absence of other predicting risk factors suggests that the Fontan state itself or the transition to it is the risk factor for such decline. ${ }^{3}$ However, the precise mechanism to account for such deterioration is unknown. While ventricular systolic function is relatively well preserved after the Fontan procedure ${ }^{45}$ diastolic dysfunction has been reported in several studies of early and medium term survivors. ${ }^{6-8}$ The acute preload reduction which occurs as a result of transition to the Fontan state leads to an increase in mass:volume ratio and inappropriate "hypertrophy" of the left ventricle. ${ }^{9}$ Coincident with this, there is evidence of impaired ventricular relaxation manifested by prolonged isovolumic relaxation time (IVRT), ${ }^{6}$ a reduction in the early rapid filling, ${ }^{6-8}$ abnormal wall motion, ${ }^{10}$ and intracavitary flow during isovolumic relaxation. ${ }^{6}$ Regression of this hypertrophy has been shown in follow up studies demonstrating a ventricular mass:volume ratio similar to that preoperatively at one to three years postoperation, ${ }^{41}$ although abnormal ventricular relaxation remains. ${ }^{7}$ Besides its deleterious effect on early diastolic filling, impaired relaxation may result in a state of reduced ventricular compliance. Thus, in hypertrophic cardiomyopathy, delayed relaxation with continued interaction of the contractile elements results in an increase in intraventricular pressure in all phases of diastolic filling. ${ }^{12}$ Fur- thermore, there is evidence that chronic reduction of preload to the left ventricle can cause reduction in compliance. ${ }^{13}$ This could have important adverse consequences were it to occur in patients after the Fontan procedure, in whom a chronic reduction of pulmonary blood flow results in long term preload reduction of the ventricle. ${ }^{14}$

This study examines prospectively Doppler indexes of left ventricular diastolic function in a cohort of patients studied in detail early and late postoperatively in order to assess any longitudinal changes associated with the Fontan circulation.

\section{Patients and method}

Thirteen subjects (seven male and six female), who underwent the Fontan operation at mean (SD) 5.5 (3.2) (range 1.5 to 12.5 ) years old, were studied at 2.8 (2.0) (early) and again 11.4 (2.0) years (late) after the operation. These subjects belonged to the cohort of 25 patients described in our previous study on incoordinate ventricular relaxation after the Fontan procedure ${ }^{6}$ Twelve patients were not available for follow up owing to death $(n=5)$, migration $(n=2)$, refusal $(n=2)$, and loss of contact $(n=3)$. Of the 13 patients restudied, nine had tricuspid atresia (with the ventriculo-arterial connection concordant in seven, discordant in one, and double outlet left ventricle in one), three had double inlet ventricle, and one had pulmonary atresia with intact ventricular septum. Before the Fontan procedure, nine 


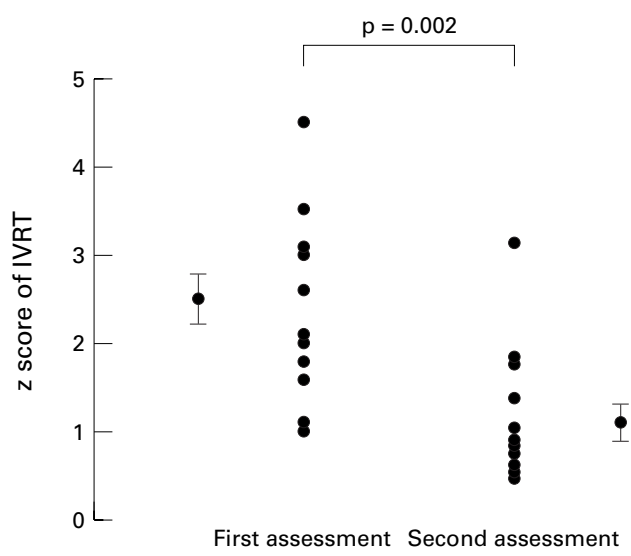

Figure 1 Vertical scatter plots of the $z$ score of the IVRT at first and second assessments. The error bar represents mean (SEM).

patients had undergone insertion of a systemic pulmonary arterial shunt and two had undergone pulmonary arterial banding. The remaining two had undergone the Fontan operation as a primary procedure. Atriopulmonary anastomosis was performed in 10 patients, total cavopulmonary connection in one, and in two the rudimentary ventricle was incorporated into the anastomosis.

DOPPLER ECHOCARDIOGRAPHIC ASSESSMENT Doppler echocardiographic assessment was performed as described in our previous study. ${ }^{6}$ Simultaneous respirometer, ECG, and phonocardiograms were recorded. All recordings were made at a paper speed of $100 \mathrm{~mm} / \mathrm{s}$. The previous recordings, obtained in identical fashion, from all 13 subjects were retrieved for reanalysis.

The R-R interval, the duration of total electromechanical systole (TEMS), IVRT, and deceleration time of the atrioventricular flow during early diastole (E wave) and atrial systole (A wave) were measured. TEMS was taken as the time interval between the onset of QRS complex and the first high frequency component of the second heart sound. IVRT was measured as the time interval between the first high frequency component of the second heart sound and the onset of early ventricular filling $\left(\mathrm{A}_{2}-\mathrm{E}\right)$. The deceleration time was measured as the time interval between the peak of the $\mathrm{E}$ or $\mathrm{A}$ wave to the intersection of the extrapolation of deceleration flow and baseline. ${ }^{15}{ }^{16}$ Peak E and A wave velocities were measured and the $\mathrm{E}: \mathrm{A}$ and $\mathrm{E}:(\mathrm{E}+\mathrm{A})$ ratios were calculated. Intraventricular diastolic flow during isovolumic relaxation and systolic flow were identified as previously described. ${ }^{6} \mathrm{Mid}$ diastolic flow was defined as antegrade atrioventricular flow occurring between the early diastolic and atrial systolic flow. ${ }^{17}$ Measurements were averaged over three cardiac cycles for all indices. Values obtained from the two assessments were compared.

STATISTICAL ANALYSIS

The data were expressed as mean (SD) unless otherwise stated. As the peak E and A wave velocities and the $\mathrm{E}: \mathrm{A}$ ratio show no age dependent changes, ${ }^{15}{ }^{18}$ they were not adjusted for age before comparison. Isovolumic relaxation time and $\mathrm{E}$ wave deceleration time are age dependent, however, and were standardised using reference values previously reported and expressed as $\mathrm{z}$ scores. ${ }^{15}$ The A wave deceleration time was not adjusted as no paediatric reference is available. However, the A wave deceleration time has been shown not to change between the ages of 20-80 years. ${ }^{16}$ The difference between the group means of early and late assessments was assessed by two tailed paired Student's $t$ test, and that between the patients and normal reference data by two tailed unpaired $t$ test. Association between the prevalence of different types of intraventricular flow and the timing of follow up was assessed by Fisher's exact test. The relation between IVRT and $\mathrm{E}$ wave velocity was assessed by linear regression analysis. A value of $p<0.05$ was considered significant.

\section{Results}

TIME INTERVALS

The R-R interval was significantly longer at follow up than at the first assessment (885 (111) $\mathrm{ms} v 723$ (109) ms, p < 0.001). In contrast, the $\mathrm{z}$ score of IVRT decreased from $+2.50(1.00)$ to $+1.24(0.80)(p=0.002)$ (fig 1 ), while the $z$ score of $E$ wave deceleration time decreased from -1.69 (1.31) to -2.40 (1.47) $(p=0.03)$. Despite the shortening of the age standardised IVRT on late follow up, it remained significantly longer than normal (table 1). In contrast, the age standardised E wave deceleration time remained persistently shorter than normal. The A wave deceleration time tended to decrease with time (early 80 (12) ms $v$ late 73 (11) ms, $\mathrm{p}=0.13$ ).

Table 1 Time intervals, and atrioventricular flow velocity and ratios, early and late after the Fontan operation

\begin{tabular}{|c|c|c|c|c|c|c|}
\hline & Early & Late & Normal reference ${ }^{15}$ & $\begin{array}{l}\text { Early } \\
\text { versus late } \\
\text { ( } p \text { value) }\end{array}$ & $\begin{array}{l}\text { Normal } \\
\text { versus early } \\
\text { ( } p \text { value) }\end{array}$ & $\begin{array}{l}\text { Normal } \\
\text { versus late } \\
\text { ( } p \text { value) }\end{array}$ \\
\hline R-R interval (ms) & $885(111)$ & $723(109)$ & - & $<0.001$ & - & - \\
\hline IVRT (ms) & $88(13)$ & $86(7)$ & - & NS & - & - \\
\hline z score of IVRT & $+2.5(1.00)$ & $+1.24(0.8)$ & $0.0(1.0)$ & 0.002 & $<0.001$ & $<0.001$ \\
\hline E wave deceleration time (ms) & $119(25)$ & $115(27)$ & - & NS & - & - \\
\hline $\mathrm{z}$ score of $\mathrm{E}$ wave deceleration time & $-1.69(1.31)$ & $-2.4(1.47)$ & $0.0(1.0)$ & 0.03 & $<0.001$ & $<0.001$ \\
\hline A wave deceleration time (ms) & $80(12)$ & $73(11)$ & - & 0.13 & - & - \\
\hline E wave velocity $(\mathrm{cm} / \mathrm{s})$ & $47(20)$ & $50(11)$ & $\begin{array}{l}92(14)(3-8 \text { years }) 88(14) \\
(13-17 \text { years })\end{array}$ & NS & $<0.001$ & $<0.001$ \\
\hline A wave velocity $(\mathrm{cm} / \mathrm{s})$ & $32(12)$ & $33(12)$ & $\begin{array}{l}42(11)(3-8 \text { years) } 39(8)(13-17 \\
\text { years) }\end{array}$ & NS & 0.003 & 0.02 \\
\hline E:A velocity ratio & $1.6(0.6)$ & $1.7(0.6)$ & $\begin{array}{l}2.4(0.7)(3-8 \text { years }) 2.3(0.6) \\
(13-17 \text { years })\end{array}$ & NS & $<0.001$ & 0.001 \\
\hline $\mathrm{E}:(\mathrm{E}+\mathrm{A})$ velocity ratio & $0.6(0.07)$ & $0.6(0.07)$ & - & NS & - & - \\
\hline
\end{tabular}




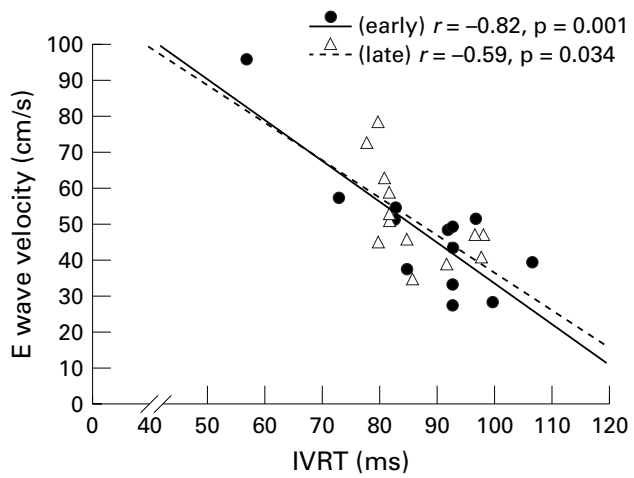

Figure 2 Scatter plot of the early diastolic flow velocity $(E$ wave velocity) against IVRT early (closed circle) and late (open triangle) after the Fontan procedure, showing a significant inverse relation.

\section{ATRIOVENTRICULAR FLOW}

Atrioventricular Doppler flow showed E and A wave filling in all the patients studied, allowing study of individual velocity profile. The $\mathrm{E}$ and $\mathrm{A}$ wave velocities, and $\mathrm{E}: \mathrm{A}$ and $\mathrm{E}:(\mathrm{E}+\mathrm{A})$ ratios were similar early and late after the Fontan procedure (table 1). However, the $\mathrm{E}$ and $\mathrm{A}$ wave velocity remained significantly lower than normal both early and late after the operation. The smaller E:A ratio in the patients resulted from the disproportionately lower $\mathrm{E}$ wave velocity. The $\mathrm{E}$ wave velocity was inversely related to IVRT both early $(r=-0.82$,

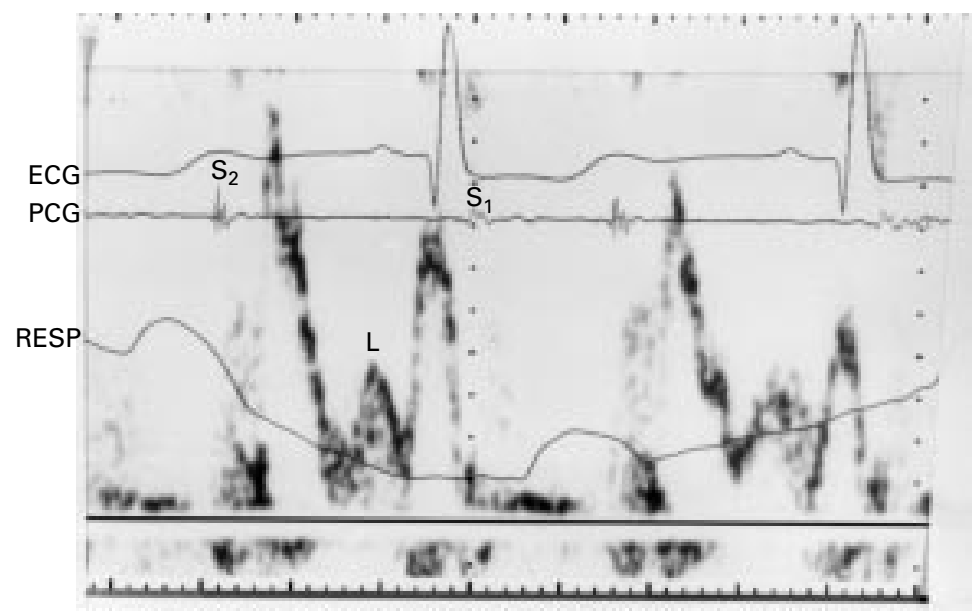

Figure 3 Pulsed wave Doppler velocity tracing with simultaneous ECG, phonocardiogram (PCG), and respirometer (RESP) in a patient assessed late after the Fontan operation. Mid diastolic antegrade flow $(L)$ is shown, which occurs between the early diastolic and atrial systolic flow. Isovolumic relaxation flow, present also in this patient, is faintly shown. $S_{1}$ first heart sound; $S_{2}$, second heart sound.

Table 2 Comparison of the follow up duration, time intervals, and atrioventricular flow profies between patients with disappearance of diastolic isovolumic relaxation (IVR) flow and those with persistent IVR flow

\begin{tabular}{llll}
\hline & $\begin{array}{l}\text { Patients with } \\
\text { disappearance of IVR } \\
\text { flow }(n=6)\end{array}$ & $\begin{array}{l}\text { Patients with } \\
\text { persistent IVR } \\
\text { flow }(n=5)\end{array}$ & p Value \\
\hline Age (years) & $17.4(3.7)$ & $16.6(2.1)$ & NS \\
Duration since operation (years) & $12.4(2.1)$ & $9.8(0.8)$ & 0.024 \\
R-R interval (ms) & $890(109)$ & $841(40)$ & NS \\
IVRT (ms) & $88(8)$ & $86(8)$ & NS \\
z score of IVRT & $+1.34(1.00)$ & $+1.01(0.54)$ & NS \\
E wave deceleration time (ms) & $106(21)$ & $124(32)$ & NS \\
z score of E wave deceleration time & $-2.77(1.21)$ & $-1.97(1.80)$ & NS \\
A wave deceleration time (ms) & $75(13)$ & $75(8)$ & NS \\
E wave velocity (cm/s) & $46(9)$ & $54(13)$ & NS \\
A wave velocity (cm/s) & $30(10)$ & $35(16)$ & NS \\
E:A velocity ratio & $1.7(0.8)$ & $1.7(0.4)$ & NS \\
\hline
\end{tabular}

$\mathrm{p}=0.001)$ and late $(r=-0.59, \mathrm{p}=0.034)$ after the operation (fig 2).

The prevalence of mid diastolic flow (fig 3) increased significantly on late follow up. It was detected in $23 \%(3 / 13)$ and $77 \%(10 / 13)$ of patients at the first and second assessment, respectively $(p=0.02)$. The flow was directed from base to apex. The flow velocity did not show any difference between the two assessments (early $20(6) \mathrm{cm} / \mathrm{s} v$ late 20 (7) cm/s, $\mathrm{p}=0.97)$. Atrioventricular valvular regurgitation, noted in only two patients, was trivial.

\section{INTRAVENTRICULAR FLOW}

The early diastolic flow, directed from base to apex, occurred during isovolumic relaxation and ended with the start of atrioventricular flow. This was found in $85 \%(11 / 13)$ of patients early after the operation, but only in $38 \%$ $(5 / 13)$ of patients on follow up $(p=0.04)$. This abnormal flow was absent in two patients during both assessments. There was no significant difference in the flow velocity between the two assessments (early 19 (8) $\mathrm{cm} / \mathrm{s} \quad v$ late 18 (6) $\mathrm{cm} / \mathrm{s}, \mathrm{p}=0.75)$. Compared with patients having persistent isovolumic flow noted at late follow up, those with disappearance of the flow had significantly longer follow up duration and tended to have shorter $\mathrm{E}$ wave deceleration time (table 2).

Systolic intraventricular flow, which began in early systole after the closure of the atrioventricular valve, was directed from base to apex. It was found in $77 \%(10 / 13)$ of patients early after the operation, and in $62 \%(8 / 13)$ patients at follow up $(\mathrm{p}=1.0)$. The duration (early 66 $(14) \% v$ late $53(14) \%$ of TEMS, p = 0.07) and velocity (early 21 (4) $v$ late 17 (5) $\mathrm{cm} / \mathrm{s}$, $\mathrm{p}=0.19)$ of the systolic flow were similar in both occasions.

\section{Discussion}

This study demonstrates changes in diastolic Doppler indices consistent with reduced compliance of the systemic ventricle and persisting abnormalities of relaxation late after the Fontan procedure. The $\mathrm{z}$ scores of IVRT and $\mathrm{E}$ wave deceleration time decreased significantly, and the A wave deceleration time tended to decrease at the late follow up assessment. Compared with normal, the $\mathrm{z}$ score of $\mathrm{E}$ wave deceleration time, $\mathrm{E}$ and $\mathrm{A}$ wave velocities, and $\mathrm{E}$ :A ratio were persistently lower, while the $\mathrm{z}$ score of IVRT was persistently higher both early and late after the operation. The prevalence of isovolumic intraventricular flow decreased while that of mid diastolic flow increased significantly on follow up.

Previous studies have shown impaired systemic ventricular relaxation early after the Fontan procedure, ${ }^{6-8}$ coincident with the increase in mass:volume ratio and acquired "hypertrophy" of the ventricle after acute preload reduction on transition to the Fontan state. ${ }^{9}{ }^{10}$ Compared with our findings early after the operation, the age standardised IVRT and prevalence of abnormal isovolumic relaxation flow decreased significantly on follow up. It is likely that regression of the ventricular mass after volume unloading results in more coordinate relaxation and 
improvement of the impaired ventricular relaxation. ${ }^{411}$ Nonetheless, the fact that the age standardised IVRT remained significantly longer and $\mathrm{E}$ wave velocity lower than normal subjects late after the operation suggests persistent impairment. This is substantiated by the significant inverse relation between IVRT and $\mathrm{E}$ wave velocity. In the light of our other findings, all of which suggest a trend towards reduced ventricular compliance, an additional mechanism may be invoked to explain the longitudinal changes.

The standardised E wave deceleration time was shorter than normal early after the operation and shortened further during follow up. Similarly, mid diastolic flow was also more prevalent. Shortening of the $\mathrm{E}$ wave deceleration time implies more rapid equalisation of the pressure between the pulmonary venous atrium and systemic ventricle, and has been demonstrated in many disease states associated with decreased ventricular compliance. ${ }^{19-21} \mathrm{Mid}$ diastolic flow arises from the re-establishment of a positive atrioventricular pressure gradient after its reversal with filling of the ventricle, the magnitude of which was increased with increased ventricular stiffness in a mathematical model, ${ }^{17}$ and has been demonstrated in adults with hypertrophic cardiomyopathy and a raised left ventricular end diastolic pressure. ${ }^{22}$ The small decrease in A wave deceleration further supports the possibility of decreased left ventricular compliance; a recent study in adults showed it to be associated with raised systemic ventricular filling pressure. ${ }^{23}$

The exact mechanism for the proposed reduction of the ventricular compliance remains speculative. The substrate for its development may exist before the Fontan procedure. An increase in cross linking of types I and III collagen occurs in experimentally induced volume loaded left ventricular hypertrophy secondary to aortocaval fistula, ${ }^{24}$ and clinical studies have shown an increased left ventricular myocardial fibrous tissue and an age related increase in subepicardial fibrosis in hearts with tricuspid atresia. ${ }^{25}$ Reduction of left ventricular compliance has also been demonstrated in mitral stenosis where the left ventricle is chronically underfilled..$^{13}$ Reduced filling of the systemic ventricle that occurs after the Fontan procedure because of a persistently low cardiac output may similarly reduce the ventricular compliance. Finally, abnormalities of ventriculo-arterial coupling may have an adverse effect. Slowing of the ventricular relaxation velocity with increasing left ventricular afterload has been shown in animal studies, and postulated to be caused by the longer time required for disengagement of the greater number of cross bridges formed. ${ }^{26}$ Increased systemic vascular resistance is characteristic in patients after the Fontan procedure, ${ }^{8}$ although it is worth noting that angiotensin converting enzyme inhibition to reduce systemic vascular resistance failed to influence Doppler filling characteristics and led to reduced cardiac response to exercise in one study. ${ }^{27}$

A potential limitation of this study is that Doppler indexes of left ventricular filling are influenced by age, ${ }^{15}$ heart rate, ${ }^{15}$ valvar regurgitation, ${ }^{28}$ and loading conditions. ${ }^{29}$ The age dependent indexes were standardised and expressed as $\mathrm{z}$ scores to allow for meaningful comparison. The mean R-R interval increases by $20 \mathrm{~ms}$ per year of age from $3-18$ years. ${ }^{15}$ The strong association between age and heart rate makes it difficult to separate their individual contribution. There may also be alterations in loading conditions. Preload reduction occurs on transition to the Fontan state, but whether progressive reduction in preload has occurred on follow up is unknown. Nonetheless, the consequence would be lengthening of the $\mathrm{E}$ wave deceleration time, rather than shortening as observed. ${ }^{29}$ Similarly, the increased systemic vascular resistance that occurs after the Fontan operation should prolong the deceleration time. $^{8}{ }^{29}$

This study provides evidence of a progressive change in systemic ventricular diastolic function after the Fontan procedure. If confirmed by formal studies of ventricular compliance, increased ventricular stiffness may contribute to a progressive increase in pulmonary venous pressure, with significant implications for pulmonary blood flow and cardiac output. This may be one of the mechanisms underlying the functional deterioration late after the Fontan procedure.

1 Driscoll DJ, Offord KP, Feldt RH, et al. Five- to fifteen-year follow-up after Fontan operation. Circulation 1992;85:46996.

2 Gentles TL, Gauvreau K, Mayer JE, et al. Functional outcome after the Fontan operation: factors influencing ate morbidity. I Thorac Cardiovasc Surg 1997;114:392403.

3 Fontan F, Kirklin JW, Fernandex G, et al. Outcome after a "perfect" Fontan operation. Circulation 1990;81:1520-36.

4 Gewillig MH, Lundstrom UR, Deanfield JE, et al. Impact of Fontan operation on left ventricular size and contractility in tricuspid atresia. Circulation 1990;81:118-27.

5 Hagler DJ, Seward JB, Tajik AJ, et al. Functional assessment of the Fontan operation: combined two-dimensional and Doppler echocardiographic studies. $7 \mathrm{Am}$ Coll Cardiol 1984;4:745-64

6 Penny DJ, Rigby ML, Redington AN. Abnormal patterns of intraventricular flow and diastolic filling after the Fontan operation: evidence for incoordinate ventricular wall motion. Br Heart $\mathcal{F}$ 1991;66:375-78.

7 Frommelt PC, Snider AR, Meliones JN, et al. Doppler assessment of pulmonary artery flow patterns and ventricuassessment of pulmonary artery flow patterns and ventricu68:1211-15.

8 Akagi T, Benson LN, Gilday DL, et al. Influence of ventricular morphology on diastolic filling performance in double-inlet ventricle after the Fontan procedure. $\mathcal{F} \mathrm{Am}$ Coll Cardiol 1993;22:1948-52.

9 Penny DJ, Lincoln C, Shore DF, et al. The early response of the systemic ventricle during transition to the Fontan circulation .... an acute hypertrophic cardiomyopathy? Cardiology in the Young 1992;2:78-84.

10 Penny DJ, Redington AN. Angiographic demonstration of incoordinate motion of the ventricular wall after the Fontan operation. Br Heart $\mathcal{7} 1991 ; 66: 456-9$.

11 Fogel MA, Weinberg PM, Chin AJ, et al. Late ventricular geometry and performance changes of functional single ventricle throughout stages Fontan reconstruction assessed by magnetic resonance imaging. $f \mathrm{Am}$ Coll Cardiol by magnetic reson.

12 Bonow RO. Left ventricular diastolic function in hypertrophic cardiomyopathy. Herz 1991;16:13-21.

13 Liu CP, Ting CT, Yang TM, et al. Reduced left ventricular compliance in human mitral stenosis. Circulation 1992;85: 1447-56.

14 Shekerdemian L, Bush A, Shore DF, et al. Cardiopulmonary interaction after Fontan operation. Augmentation of cardiac output using negative pressure ventilation. Circulation 1997;96:3934-42.

15 O'Leary PW, Durongpisitkul K, Cordes TM, et al. Diastolic ventricular function in children: a Doppler echocardiographic study establishing normal values and predictors of increased ventricular end-diastolic pressure. Mayo Clin increased ventricular
Proc 1998;73:616-28.

16 Mantero A, Gentile F, Gualtierotti C, et al. Left ventricular diastolic parameters in 288 normal subjects from 20 to 80 diastolic parameters in 288 normal subject
years old. Eur Heart $\mathcal{f}$ 1995;16:94-105. 
17 Keren G, Meisner JS, Sherez J, et al. Interrelationship of mid-diastolic mitral valve motion, pulmonary venous flow, Circulation 1986:74:36-44.

18 Schmitz L, Koch H, Bein G, et al. Left ventricular diastolic function in infants, children, and adolescents. Reference values and analysis of morphologic and physiologic determinants of echocardiographic Doppler flow signals during growth and maturation. $\mathcal{F}$ Am Coll Cardiol 1998;32: 1441-8.

19 Pinamonti B, Di Lenarda A, Sinagra G, et al. Restrictive left ventricular filling pattern in dilated cardiomyopathy assessed by Doppler echocardiography: clinical, echocardiographic and hemodynamic correlations and prognostic implications. F Am Coll Cardiol 1993;22:808-15.

20 Appleton CP, Hatle LK, Popp RL. Differentiation of restrictive ventricular physiology by Doppler echocardiography. $7 \mathrm{Am}$ Coll Cardiol 1988;11:757-68.

21 Nishimura RA, Abel MD, Hatle LK, et al. Assessment of diastolic function of the heart: background and current diastolic function applications of Doppler echocardiography.

22 Oki T, Fukuda N, Iuchi A, et al. Evaluation of left ventricular diastolic hemodynamics from the left ventricular inflow lar diastolic hemodynamics from the left ventricular inflow
and pulmonary venous flow velocities in hypertrophic cardiomyopathy. Fpn Heart $\mathcal{F}$ 1995;36:617-27.
23 Tenenbaum A, Motro M, Hod H, et al. Shortened Dopplerderived mitral A wave deceleration time: an important prefilling pressure. $7 \mathrm{Am}$ Coll (00-5.

24 Iimoto DS, Covell JW, Harper E. Increase in cross-linking of type I and type III collagens associated with volume-loaded hypertrophy. Circ Res 1988;63:399-408.

25 Ho SY, Jackson M, Kilpatrick L, et al. Fibrous matrix of ventricular myocardium in tricuspid atresia compared with normal heart. A quantitative analysis. Circulation 1996;94: 1642-6.

26 Gaasch WH, Blaustein AS, Andrias CW, et al. Myocardial relaxation. II. Hemodynamic determinants of rate of left ventricular isovolumic pressure decline. Am $\mathcal{F}$ Physio 1980; 239:H1-6.

27 Kouatli AA, Garcia JA, Zellers TM, et al. Enalapril does not enhance exercise capacity in patients after Fontan procedure. Circulation 1997;96:1507-12.

28 Takenaka K, Dabestani A, Gardin JM. Pulsed Doppler echocardiographic study of left ventricular filling in dilated echocardiographic study of left ventricular filling

29 Nishimura RA, Tajik AJ. Quantitative hemodynamics by Nishimura RA, Tajik AJ. Quantitative hemodynamics by
Doppler echocardiography: a noninvasive alternative to cardiac catheterization. Prog Cardiovasc Dis 1994;36:309

\section{Ventricular fibrillation provoked by cardioversion and asynchronous pacing}

A 57 year old women with sick sinus syndrome had been implanted with a Biotronik Pikos LP VVI pacemaker. Sinus rhythm was successfully restored by antiarrhythmic drugs three times during the past year. Because of recurrence of atrial fibrillation she was on permanent anticoagulant treatment. A recent attack of atrial fibrillation did not respond to antiarrhythmic treatment, therefore we planned a direct current cardioversion. The pacemaker was programmed to VOO mode with 50 beats/min frequency. The figure shows atrial fibrillation with high ventricular rate, as well as asynchronous pacing. No ventricular capture could be seen, because the pacemaker spikes were falling on the refractory period. The third pacemaker spike-which coincided with the beginning of the $T$ wave-triggered the synchronisation of the cardioverter, resulting in ventricular fibrillation; she underwent immediate defibrillation.

The VOO mode or use of a magnet are recommended for protective purposes during certain interventions, like lithotripsy or electrocautery. Be cautious, however, as the VOO mode should be avoided during direct current cardioversion - as with our patient, the false synchronisation of the cardioverter may result in ventricular fibrillation.

ADAM BOHM ADAM SZEKELY ISTVÁN PRÉDA

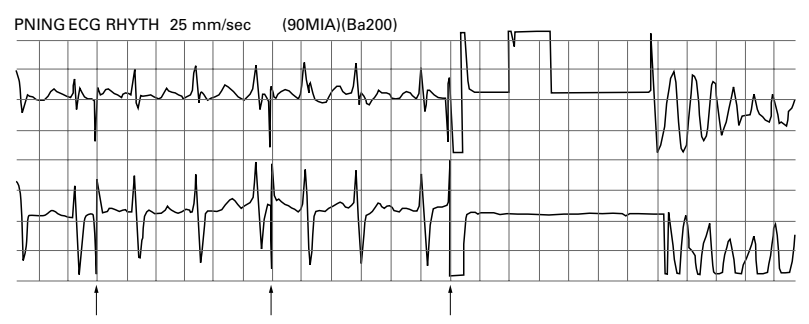

